Bryn Mawr College

Scholarship, Research, and Creative Work at Bryn Mawr

College

Physics Faculty Research and Scholarship

Physics

2015

\title{
Nonexponential 1H Spin-Lattice Relaxation and Methyl Group Rotation in Molecular Solids
}

Peter A. Beckmann

Bryn Mawr College, pbeckman@brynmawr.edu

Let us know how access to this document benefits you.

Follow this and additional works at: http://repository.brynmawr.edu/physics_pubs

Part of the Physics Commons

\section{Custom Citation}

Nonexponential $1 \mathrm{H}$ spin-lattice relaxation and methyl group rotation in molecular solids. P. A. Beckmann 2015 Solid State Nuclear Magnetic Resonance 71 91-95.

This paper is posted at Scholarship, Research, and Creative Work at Bryn Mawr College. http://repository.brynmawr.edu/physics_pubs/90

For more information, please contact repository@brynmawr.edu. 


\section{Nonexponential ${ }^{1} \mathrm{H}$ Spin-Lattice Relaxation and Methyl Group Rotation in Molecular Solids}

Peter A. Beckmann, Department of Physics, Bryn Mawr College, Bryn Mawr, PA 19010, USA

Corresponding Author: Peter Beckmann (610-526-5358; pbeckman@brynmawr.edu)

2015 Solid State Nuclear Magnetic Resonance 71 91-95

keywords: methyl rotation; nonexponential spin-lattice relaxation; molecular solds

We report a quantitative measure of the nonexponential ${ }^{1} \mathrm{H}$ spin-lattice relaxation resulting from methyl group $\left(\mathrm{CH}_{3}\right)$ rotation in six polycrystalline van der Waals solids. We briefly review the subject in general to put the report in context. We then summarize several significant issues to consider when reporting ${ }^{1} \mathrm{H}$ or ${ }^{19} \mathrm{~F}$ spin-lattice relaxation measurements when the relaxation is resulting from the rotation of a $\mathrm{CH}_{3}$ or $\mathrm{CF}_{3}$ group in a molecular solid.

Introduction

In 1964, Runnells [1] and, independently, Hilt and Hubbard [2] showed that the nuclear spinlattice relaxation resulting from the modulation of the spin-spin interactions among the three spin$1 / 2{ }^{1} \mathrm{H}$ nuclei in a $\mathrm{CH}_{3}$ (methyl) group [or among the three spin- $1 / 2{ }^{19} \mathrm{~F}$ nuclei in a $\mathrm{CF}_{3}$ (fluoromethyl) group] was inherently nonexponential. A decade later a slightly different approach to the problem, also arriving at the fact that the relaxation was nonexponential, was suggested [3]. This phenomenon is often neglected, which means that, knowingly or unknowingly, an average relaxation rate is reported. Here, following a brief review of this subject, we pull together results from six polycrystalline van der Waals organic solids with quite different methyl group environments and quite different motions of the methyl group rotation axes, to show that this phenomenon is ubiquitous. We also show that the relaxation at lower temperatures, though always reported as being exponential within experimental uncertainty, can, in a statistical sense when many experiments are considered, be seen also to be slightly nonexponential.

\section{Background}

Nuclear spin-lattice relaxation for an ensemble of isolated interacting pairs of spin- $1 / 2$ nuclei whose internuclear vectors are of fixed length and are reorienting isotropically and randomly is presented by Abragam [4]. The spin-lattice relaxation rate for this two-spin, four-state system is strictly exponential. Abragam reviews developments prior to about 1960, including the important contributions from Bloembergen, Purcell and Pound [5], Wangsness and Bloch [6], Solomon [7], 
Bloch [8], and Hubbard [9]. Additional important contributions in this early period were made by Woessner [10], Bloch [11], Redfield [12], Tomita [13], and Stejskal and Gutowsky [14]. Later developments along with appropriate references appear in the texts by Slichter [15], Ernst $e t$ al.[16], and Kimmich [17]. Goldman has produced a review of the formalism [18]. In this model, traditionally referred to as the BPP model [5], a perturbed bulk spin-1/2 nuclear magnetization returns to its equilibrium value exponentially with the spin-lattice relaxation rate $R=A[J(\omega, \tau)+$ $4 J(2 \omega, \tau)]$ with $A=B\left(\mu_{0} / 4 \pi^{2}\right)^{2}\left(\hbar \gamma^{2} / r^{3}\right)^{2}, B=3 / 20, J(\omega, \tau)=2 \tau /\left(1+\omega^{2} \tau^{2}\right)$, and $\tau=\tau_{\infty}$ $\exp \left(E_{\mathrm{NMR}} / k T\right)$ [4]. Here $\mu_{0}$ is the magnetic constant, $\gamma$ is the ${ }^{1} \mathrm{H}$ (or ${ }^{19} \mathrm{~F}$ ) magnetogyric ratio, $r$ is the constant $\mathrm{H}-\mathrm{H}$ (or F-F) distance characterizing the isolated randomly and isotropically orienting spin-1/2 pairs, $J(\omega, \tau)$ is the spectral density (the frequency spectrum of the local time-dependent magnetic fields), $\omega$ is the NMR angular frequency, $\tau$ is the correlation time which can be taken to be the mean resident time between hops in a Poisson classical hopping process [14, 19-26], $\tau_{\infty}$ is a preexponential factor $[19,27,28]$, and $E_{\mathrm{NMR}}$ is an NMR activation energy which is closely related to a barrier the spin pair must overcome to reorient.

The nuclear spin-lattice relaxation resulting from the reorientation of a $\mathrm{CH}_{3}$ or $\mathrm{CF}_{3}$ group involves a three spin-1/2 system and the BPP model presented above does not directly apply. First, the three spins give rise to eight spin states [1-3, 29, 30]. Second, in a solid, each triangle of spins reorients randomly (on the NMR time scale for the cases considered here) but in a plane, not isotropically. One of the examples presented here involves the methyl group rotation axis also reorienting randomly (but not isotropically) on the NMR time scale. Third, the motion of the three spin-spin vectors is 100\% correlated. Runnels [1] and Hilt and Hubbard [2] dealt with these complications in detail and the result for an ensemble of isolated $\mathrm{CH}_{3}$ or $\mathrm{CF}_{3}$ groups whose rotation axes are oriented in the same direction (with respect to the applied magnetic field) is that the relaxation proceeds as the sum of four exponentials. The recovery to equilibrium of a perturbed ${ }^{1} \mathrm{H}$ or ${ }^{19} \mathrm{~F}$ magnetization can be expressed in algebraic form as a function of the angle $\alpha$ between the $\mathrm{CH}_{3}$ or $\mathrm{CF}_{3}$ group rotation axis and the applied magnetic field. Although no relaxation experiment could ever observe four exponentials (which would involve at least nine adjustable parameters), nonexponential relaxation has been observed in a single crystal of $\mathrm{CF}_{3} \mathrm{COOAg}$ where the crystal structure is such that all $\mathrm{CF}_{3}$ rotation axes are parallel [31-33]. Hilt and Hubbard [2] performed a numerical averaging over all orientations of the $\mathrm{CH}_{3}\left(\right.$ or $\left.\mathrm{CF}_{3}\right)$ group 
rotation axes appropriate for a polycrystalline powder and found that the relaxation is still nonexponential, particularly near the relaxation rate maximum $(\omega \tau \sim 1)$ and at higher temperatures $(\omega \tau<1)$. Nonexponential spin-lattice relaxation has been observed in polycrystalline solids [34-41] in temperature regions corresponding to $\omega \tau \sim 1$ and $\omega \tau<1$. All these studies report exponential relaxation (within experimental uncertainty) at lower temperatures $(\omega \tau>1)$.

Early experiments where nonexponentiality was observed were performed on solid samples made of small molecules (compared with those reported here). In these samples, motions, like whole-molecule tumbling in the solid state, are sometimes occurring on the NMR timescale in addition to methyl group rotation $[34,38,40,42]$. The degree to which the relaxation is nonexponential depends on the relative time scales of the two motions (methyl group rotation and molecular tumbling) as well as on the geometry of the molecule [43]. The presence of either ${ }^{1} \mathrm{H}$ ${ }^{1} \mathrm{H}$ spin-spin interactions between methyl group protons and other protons or between protons on different methyl groups makes the relaxation more exponential [34, 37, 44]. This has been born out in experiments with solids comprised of larger organic molecules with several or many static (on the NMR time scale) $\mathrm{H}$ atoms. In many of these cases, the departure from exponential relaxation (at all temperatures) is very slight or not observed at all [45-48].

The nonexponential relaxation discussed here should not be confused with other origins of nonexponential relaxation. Nonexponential nuclear spin-lattice relaxation can also result from both (1) a distribution of correlations times [because of a distribution of $E_{\mathrm{NMR}}$ values in $\tau=\tau_{\infty}$ $\left.\exp \left(E_{\mathrm{NMR}} / k T\right)\right]$ in the case of limited spin diffusion or (2) because of an inherently nonexponential correlation function, that is $\tau \neq \tau_{\infty} \exp \left(E_{\mathrm{NMR}} / k T\right)[49,50]$. The latter nonPoisson process usually originates from a time-ordered (bottleneck) process (A can't move until B moves [51]). In some cases, one can distinguish between these two cases [50]. But for $\mathrm{CH}_{3}$ or $\mathrm{CF}_{3}$ rotation in the classical hopping limit, the dynamics are strictly Poissonian. The relaxation rates are distributed because the ${ }^{1} \mathrm{H}$ or ${ }^{19} \mathrm{~F}$ bulk magnetization recoveries $d M(\alpha) / d t$ are distributed. The recoveries $d M(\alpha) / d t$ are distributed because the angle $\alpha$ between the $\mathrm{CH}_{3}$ or $\mathrm{CF}_{3}$ group rotation axis and the applied magnetic field are distributed [1,2]. The correlation time $\left.\tau=\tau_{\infty} \exp \left(E_{\mathrm{NMR}} / k T\right)\right]$ is still the same for every $\mathrm{CH}_{3}$ or $\mathrm{CF}_{3}$ group so long as all $\mathrm{CH}_{3}$ or $\mathrm{CF}_{3}$ groups have the same environment as is the case for five of the six examples presented in this study. This does mean that the averaging 
should be performed over the magnetizations $M(\alpha)$ and not the relaxation rates $R(\alpha)$ contained in $d M(\alpha) / d t$ [52-54].

The nonexponential relaxation resulting from $\mathrm{CH}_{3}$ or $\mathrm{CF}_{3}$ group rotation in polycrystalline samples can be adapted to the isolated two-spin relaxation rate model presented above. That is, the BPP model can be used with the two-spin $B=3 / 20$ presented above replaced with $B=(9 / 40)(n / N)$ [14] where $n$ is the number of $\mathrm{H}$ or $\mathrm{F}$ atoms in equivalent methyl (or fluoromethyl) groups and $N$ is the number of $\mathrm{H}$ or $\mathrm{F}$ atoms in the molecule [55-57]. This does assume that spin diffusion is rapid enough that a common spin temperature is attained in a time short compared with the spin-lattice relaxation time (the inverse of the spin-lattice relaxation rate). To use this revised BPP algebraic expression, however, the initial slope of the nonexponentially relaxing magnetization must be determined $[2,58]$. We call this initial rate $R_{\mathrm{S}}[59]$. At short times, the perfect correlations between the three $\mathrm{H}-\mathrm{H}$ vectors (or between the three $\mathrm{F}-\mathrm{F}$ vectors) do not play a role. This shorttime rate $R_{\mathrm{S}}$ is always larger than some average rate [59]. Measuring $R_{\mathrm{S}}$ is time consuming and imprecise. The signal-to-noise needs be quite good to determine $R_{\mathrm{S}}$ within $20 \%$ or so. However, with the revised BPP model $R_{\mathrm{S}}=A_{\text {intra }}[J(\omega, \tau)+4 J(2 \omega, \tau)]$ with $A_{\text {intra }}=(9 / 40)(n / N)\left(\mu_{0} / 4 \pi^{2}\right)^{2}(\hbar$ $\left.\gamma^{2} / r^{3}\right)^{2}, J(\omega, \tau)=2 \tau /\left(1+\omega^{2} \tau^{2}\right)$ and $\tau=\tau_{\infty} \exp \left(E_{\mathrm{NMR}} / k T\right)$, the $\mathrm{CH}_{3}$ or $\mathrm{CF}_{3}$ group becomes a very useful probe of its intramolecular and intermolecular environment. The subscript 'intra' on $A_{\text {intra }}$ means only the intra $\mathrm{CH}_{3}$ (or $\mathrm{CF}_{3}$ ) spin-spin interactions are involved. In this case $E_{\mathrm{NMR}}$ can be related to the height of the barrier for $\mathrm{CH}_{3}$ or $\mathrm{CF}_{3}$ rotation $[58,60-63]$. In practice the parameter $A_{\text {intra }}$ in the above expression is replaced by $A_{\text {intra }}(1+y)$ where $y$, which is usually between 0 and 0.3 , is a measure of the relaxation resulting from the modulation of vectors between ${ }^{1} \mathrm{H}$ spins in a $\mathrm{CH}_{3}$ group and ${ }^{1} \mathrm{H}$ spins not in a $\mathrm{CH}_{3}$ group (or at least not in the same $\mathrm{CH}_{3}$ group). These additional interactions can be of either intramolecular of intermolecular origin. Although there are many such interactions in the system, the ${ }^{1} \mathrm{H}-{ }^{1} \mathrm{H}$ interaction falls off as $r^{-6}$ and these vectors undergo limited angular variation as a methyl group rotates. In practice, the parameter $y$ must be determined experimentally.

Fitting the nonexponential relaxation

In a relaxation experiment, exponential relaxation can be characterized by a ${ }^{1} \mathrm{H}$ or ${ }^{19} \mathrm{~F}$ bulk nuclear magnetization $M(t)=M(\infty)[1-(1-\cos \theta) \exp (-R t)] . R$ is the spin-lattice relaxation rate, $M(\infty)$ is the equilibrium magnetization, and the adjustable parameter $\theta$ accounts for imperfections in the 
perturbing pulse (or pulses in a sequence used to suppress artifacts). For an inversion-t-measure sequence, ideally $\theta=\pi$ and $(1-\cos \theta)=2$. For a saturation- $t$-measure sequence, ideally $\theta=\pi / 2$ and $(1-\cos \theta)=1 .[M(0)$, the initial magnetization after the perturbation, could be used as a fitting parameter instead of $\theta$.] We note that in these experiments, exponential relaxation involves a three-parameter fit: $R, \theta[$ or $M(0)]$, and $M(\infty)$. It should not be assumed that $M(0)=0(\theta=\pi / 2)$ for a saturation- $t$-measure sequence or that $M(0)=-M(\infty)(\theta=\pi)$ for an inversion-recovery sequence. The parameter $\theta$ or $M(0)$ should still be an adjustable parameter to eliminate the possibility of making significant systematic errors in fitting parameters [64].

Often, nonexponential relaxation is fitted using a double exponential $M(t)=M_{1}(\infty)[1-(1-$ $\left.\cos \theta) \exp \left(-R_{1} t\right)\right]+M_{2}(\infty)\left[1-(1-\cos \theta) \exp \left(-R_{2} t\right)\right]$. But this is a five-parameter fit and may be unwarranted unless a relevant model suggests such a description is appropriate. (For example, a double exponential is sometimes both required by theory and confirmed by experiment if there are both ${ }^{19} \mathrm{~F}$ and ${ }^{1} \mathrm{H}$ spins in the system [65]). Indeed, a standard non-linear fitting routine will often return reasonable uncertainties for the two rates $R_{1}$ and $R_{2}$ but on closer inspection the two magnetizations $M_{1}(\infty)$ and $M_{2}(\infty)$ will often have very large uncertainties, large enough to render the fit quite meaningless even though a visual inspection suggests the fit is acceptable. This indicates that a four parameter fit is more desirable.

One can probably invent many four-parameter functions that will fit the magnetization recovery curve in a nonexponential process. A stretched exponential $M(t)=M(\infty)[1-(1-$ $\left.\cos \theta) \exp \left\{-\left(R^{*} t\right)^{\beta}\right\}\right]$ is perhaps the most popular phenomenological model; it mimics a continuous distribution of time dependent magnetizations or relaxation rates (regardless of the physical origin) and involves only one parameter in addition to the three needed for exponential relaxation. (See [66] for a different four-parameter model appropriate for some translational diffusion processes.) We have provided a brief review of the stretched exponential (or Kohlrausch [67]) function and its use in several fields of experimental science [59]. We add two references here [68, 69] not found in our minireview [59] and we draw attention to a brief historical note concerning the history of the early use of this function in the physical sciences [70]. The characteristic relaxation rate $R^{*}$ and the stretching parameter $\beta$ in the stretched exponential function have no meaningful interpretation for NMR relaxation experiments in terms of an algebraic model for the dynamics. (The relaxation rate $R_{\mathrm{S}}$ characterizing the initial decay is the parameter that is modeled by the 
modified BPP expressions.) The stretched exponential is being used to phenomenologically mimic a distribution of decaying magnetizations or relaxation rates (see references in [59]). In the current work we use the parameter $\beta$ in the stretched exponential function in a purely phenomenological manner solely to indicate, quantitatively, the degree of nonexponentiality. As an important aside, we have determined experimentally that $R^{*}$ versus $T^{-1}$ at high temperatures $(\omega \tau<<1)$ gives the same value of the NMR activation energy $E_{\mathrm{NMR}}$ in $\tau=\tau_{\infty}$ $\exp \left(E_{\mathrm{NMR}} / k T\right)$ as does $R_{\mathrm{S}}$ versus $T^{-1}$ at high temperatures $(\omega \tau<<1)[59,71] . R^{*}$ and $\beta$ can be determined quickly and accurately. So measuring $R^{*}$ in the stretched exponential can be quite useful if the goal is to quickly obtain an NMR activation energy. The parameters $\tau_{\infty}$ and $y$ cannot be determined this way; this will lead to large systematic errors. As another aside, the best way to determine if a five-parameter double exponential fit is justified, is to first determine that a fourparameter continuous distribution fit fails. This is indeed the case at low temperatures $(\omega \tau>1)$ when there is considerable crosstalk between ${ }^{1} \mathrm{H}$ and ${ }^{19} \mathrm{~F}$ spins [65]. In this report we do not consider the case where both ${ }^{1} \mathrm{H}$ and ${ }^{19} \mathrm{~F}$ spin species are present and interacting via the dipoledipole interaction, though some of the points made here are relevant in this case in the high temperature regime $\omega \tau<1[65]$.

The experiments and their results

The parameter $\beta$ in $M(t)=M(\infty)\left[1-(1-\cos \theta) \exp \left\{-\left(R^{*} t\right)^{\beta}\right\}\right]$ is used solely to indicate the degree of nonexponentiality and has no fundamental role in comparing data with theoretical models, at least for nuclear spin-lattice relaxation experiments. Having said that, however, $\beta<1$ can be used as an indication that $\mathrm{CH}_{3}$ or $\mathrm{CF}_{3}$ group rotation may be involved in the relaxation process. Here we present the temperature dependence of $\beta$ at an NMR frequency of $\omega / 2 \pi=22.5 \mathrm{MHz}$ in six van der Waals molecular solids (1-6 in the Table) with a variety of local $\mathrm{CH}_{3}$ group environments. The relatively low NMR frequency is needed to make accessible both (1) the low temperature long correlation time limit $\omega \tau>>1$ (which must involve temperatures above those where quantum mechanical tunneling [72] may play a role) and (2) the high temperature short correlation time limit $\omega \tau<<1$ (which must include temperatures below the melting point). The parameter $\beta$ versus $T^{-1}-T^{-1}{ }_{\max }$ for 1-6 is shown in the figure. $T$ is the temperature and $T_{\max }$ is the temperature of the relaxation rate maximum where $\omega \tau \sim 1$ for methyl group rotation. The parameter $10^{-3} T^{-1}{ }_{\max }$ (in 
$\mathrm{K}^{-1}$ ) for each compound is indicated in the legend in the figure. (There are two closely spaced $T_{\max }$ for two inequivalent types of methyl groups in $\mathbf{6}$ and the value indicated in the figure legend is between the two.) The solid horizontal line in the figure indicates $\beta=1$ (exponential relaxation). However, magnetization versus time plots will always involve some noise, the fitted value of $\beta$ will always involve an uncertainty (see the figure), and our experience is that even when the signal-to-noise is large, when $\beta>0.95$, the nonexponentiality is very difficult to observe, meaning that $R^{*}$ (the stretched exponential characteristic relaxation rate) $=R_{\mathrm{S}}$ (the initial decay) $=R$ (the rate for exponential relaxation) to within experimental uncertainty. The dashed horizontal line in the figure indicates $\beta=0.95$. In four of the six van der Waals solids discussed here $(\mathbf{1}, \mathbf{4}, \mathbf{5}, \mathbf{6})$ only a single $R$ which we would now call an average relaxation rate $R_{\text {ave }}$ was originally reported $[45,73,74]$. Fortunately, $R_{\text {ave }}$ as a function of $T^{1}$ for $\omega \tau<<1$, like $R^{*}$ as a function of $T^{1}$ for $\omega \tau$ $<<1$ results in correct NMR activation energies $E_{\mathrm{NMR}}[59]$. (Although there may be sound underlying theoretical reasons for this, we rather suspect this to be more good luck than good management.) Experiments in those four samples have been repeated for this report. Details of the experimental procedure, temperature control, and temperature measurement can be found elsewhere [59,71]. The new $R_{\mathrm{S}}$ and $R^{*}$ versus $T^{1}$ plots are not presented here. Examples can be seen in the plots for compounds 2 [71] and 3 [59]. Indeed, in the figure, $\beta$ versus $T^{-1}$ for $\mathbf{2}$ is taken from [71] and $\beta$ versus $T^{-1}$ for 3 is taken from [59]. For $R_{\mathrm{S}}$ and $R^{*}$ versus $T^{1}$ for the six solids, the lowest temperatures used in the experiments were $98 \mathrm{~K}$ for $\mathbf{1}, 122 \mathrm{~K}$ for $\mathbf{2}, 91 \mathrm{~K}$ for $\mathbf{3}, 102 \mathrm{~K}$ for $\mathbf{4}$, $109 \mathrm{~K}$ for $\mathbf{5}$, and $127 \mathrm{~K}$ for $\mathbf{6}$. These temperatures are well above the temperature where methyl group tunneling need be considered [20-26, 63, 75-78]. Alternatively, the NMR activation energies for methyl group rotation (indicated in the table) are all well above $6-8 \mathrm{~kJ} \mathrm{~mol}^{-1}$, a slightly different but nevertheless related condition for not needing to consider tunneling [58].

One of the samples investigated here (1) involves a "lone" methyl group on an aromatic ring (the $\mathrm{CD}_{3}$ group in $\mathbf{1}$ is not "seen" in an ${ }^{1} \mathrm{H}$ NMR experiment and is too far away to have any effect on the $\mathrm{CH}_{3}$ group), two samples ( $\mathbf{2}$ and $\mathbf{3}$ ) have the methyl group(s) in a methoxy group(s) $\mathrm{OCH}_{3}$, one (4) has the methyl group in an ethyl group $\mathrm{CH}_{2} \mathrm{CH}_{3}$, one (5) has two methyl groups in an isopropyl group $\mathrm{CH}\left(\mathrm{CH}_{3}\right)_{2}$, and one (6) has three methyl groups in a $t$-butyl group $\mathrm{C}\left(\mathrm{CH}_{3}\right)_{3}$. These compounds provide a set of quite different intramolecular and intermolecular environments for the methyl groups. X-ray structures have been determined for five of the six samples (1 [79], 2 [71], $\mathbf{3}$ [80], $\mathbf{5}$ [81], and $\mathbf{6}$ [74]). In $\mathbf{1}$ and $\mathbf{2}$, there is one methyl group per molecule and $Z^{\prime}=1$, 
meaning that all molecules and therefore all methyl groups have the same environment (that is, they are equivalent and only one correlation time $\tau$ is needed). There are two methyl groups in $\mathbf{3}$ (one at each end of the molecule) but here $Z^{\prime}=1 / 2$, so, again, all methyl groups are equivalent. The two methyl groups in $\mathbf{5}$, though not identical (as a consequence of intermolecular interactions), have environments so similar that an NMR relaxation experiment would never detect the difference [81]. $Z^{\prime}=1$ in $\mathbf{6}$ so here all $t$-butyl groups are equivalent. However, the in-plane methyl group in the $t$-butyl group has a higher barrier $\left(24 \mathrm{~kJ} \mathrm{~mol}^{-1}\right)$ than the two out-of-plane methyl groups $\left(14 \mathrm{~kJ} \mathrm{~mol}^{-1}\right)$ as indicated in the table. This structural information is important because fitting the temperature and frequency dependence of relaxation rate data for 1-3, and 5 means there is only a single $\tau$ in $R=A_{\text {intra }}(1+y)[J(\omega, \tau)+4 J(2 \omega, \tau)]$, with $J(\omega, \tau)=2 \tau /\left(1+\omega^{2} \tau\right.$ ${ }^{2}$ ) and $\tau=\tau_{\infty} \exp \left(E_{\mathrm{NMR}} / k T\right)$, and therefore a single value of $E_{\mathrm{NMR}}, y$, and $\tau_{\infty}$. Although we have no $X$-ray data for $\mathbf{4}$, we assume that $Z^{\prime}=1$ which is consistent with the relaxation rate data (this work and [45]). Ab initio electronic structure calculations in clusters of molecules based on the X-ray diffraction structure show, in agreement with the NMR relaxation experiments, that methoxy group rotation over a barrier in $\mathbf{2}$ [71] and in $\mathbf{3}$ [80], and isopropyl group rotation over a barrier in 5 [81] is quenched by intermolecular interactions in the solid state as a consequence of the rotational asymmetry of these groups. Librations (rotational vibrations) of these groups (which are very fast on the NMR time scale) over a small angle [71, 80,81] play no role in the nuclear spin-lattice relaxation process other than adding a time dependence to the already present spatial dependence of methyl group rotation axes. No calculations have been done for $\mathbf{4}$ but we assume, as shown by the NMR relaxation experiments [ 45 and this work] that this is also the case for ethyl group rotation. In $\mathbf{6}$, there is one $\tau$ for the $t$-butyl group and its in-plane methyl group (as they both rotate in a geared manner by the plane of the aromatic ring) and another $\tau$ for the two out-of-plane methyl groups. The details of the model that characterize the superimposed motion of a $t$-butyl group and its three methyl groups [with all four motions on the NMR time scale (even if the $\tau$ s are slightly different)] is complicated $[74,83]$ but that does not concern us here. We simply want to note the fact that in this case the $t$-butyl group is rotating on the NMR time scale which means the three methyl group axes are rotating randomly (though not isotropically) on the NMR time scale and this results in the relaxation being more exponential (see the figure). 


\section{Discussion}

We provide the following conclusions concerning the interpretation of ${ }^{1} \mathrm{H}$ or ${ }^{19} \mathrm{~F}$ NMR spin-lattice relaxation experiments in polycrystalline samples composed of van der Waals molecular solids with $\mathrm{CH}_{3}$ or $\mathrm{CF}_{3}$ groups (but not both). (1) In many cases, the nonexponential recovery of a perturbed nuclear magnetization is very adequately fitted by a stretched exponential $M(t)=$ $M(\infty)\left[1-(1-\cos \theta) \exp \left\{-\left(R^{*} t\right)^{\beta}\right\}\right]$ and this is simply a phenomenological (but quantitative) way to investigate the nonexponential relaxation that involves only one parameter in addition to the fit $M(t)=M(\infty)[1-(1-\cos \theta) \exp (-R t)]$ for exponential relaxation. The parameter $\beta=1$ for exponential relaxation. (2) Fitting the nuclear magnetization recovery data with a double exponential is not justified unless the uncertainties in all five fitting parameters are reasonable and the data cannot be fitted with a four-parameter relaxation function. There are likely many four-parameter functions that will adequately characterize nonexponential relaxation; the stretched exponential function is one. (3) If the relaxation is nonexponential, the initial slope of the recovery of a perturbed magnetization is characterized by a relaxation rate $R_{\mathrm{S}}$ that can be modeled by a modified BPP theory. In this case, the $\mathrm{CH}_{3}$ or $\mathrm{CF}_{3}$ group becomes a potentially useful quantitative observer of its local environment. (4) When $\beta$ in $M(t)=M(\infty)\left[1-(1-\cos \theta) \exp \left\{-\left(R^{*} t\right)^{\beta}\right\}\right]$ is plotted as a function of the departure of the inverse temperature from the value at which the relaxation rate maximum occurs (see the figure), the temperature dependence of $\beta$ has a similar shape for all compounds so long as the $\mathrm{CH}_{3}$ or $\mathrm{CF}_{3}$ rotation axis is not moving on the $\mathrm{NMR}$ time scale (as is the case for 6 ). (5) In compounds 1-5 investigated here, methyl group rotation is the only motion on the NMR time scale and the systematic differences among the five sets of $\beta$ versus $T^{-1}$ data in the figure is due to the role of methyl - nonmethyl ${ }^{1} \mathrm{H}$ spin-spin interactions (both intramolecular and intermolecular). The stronger these interactions are (relative to the intramethyl ${ }^{1} \mathrm{H}-{ }^{1} \mathrm{H}$ interactions), the more exponential the relaxation. (6) The values of $\beta$ are statistically significantly below $\beta=1$ at low temperatures ( $\omega \tau>>1$; well below the relaxation rate maximum) where exponential relaxation is always reported. At the same time, the values of $\beta$ all have $\beta>0.95$ which we conclude to mean that reporting exponential relaxation within experimental uncertainty is justified. What this means quantitatively is that the uncertainties in $R$ in $M(t)=M(\infty)[1-(1-$ $\cos \theta) \exp (-R t)]$ (where $R$ really means an average relaxation rate $R_{\text {ave }}$ ) and $R^{*}$ in $M(t)=M(\infty)[1-$ $\left.(1-\cos \theta) \exp \left\{-\left(R^{*} t\right)^{\beta}\right\}\right]$ overlap considerably and $R=R^{*}$ to within experimental uncertainty. In 
turn, these rates will not differ within experimental uncertainty from the initial rate of relaxation

$R_{\mathrm{S}}$, the latter being the parameter that corresponds to appropriately revised BPP theory. (7) Even though $R^{*}$ cannot be adequately modeled, $\ln R^{*}$ versus $T^{-1}$ for $\omega \tau<<1$ provides an accurate determination of the NMR activation energy $E_{\mathrm{NMR}}$ (but not other parameters). This is relevant because $R^{*}$ (and $\beta$ ) can be determined quickly and accurately. (8) Compound 6 has methyl group rotation superimposed on $t$-butyl group rotation. This superimposed motion significantly reduces the degree of nonexponentiality. Indeed, the relaxation in the original work with $\mathbf{6}$ was reported as exponential within experimental uncertainty at all temperatures. Alas, here, $\beta>0.93$ over the entire temperature range which incorporates both the low $\omega \tau>>1$ and high $\omega \tau<<1$ temperature limits. However, from a statistical perspective, that is by looking at the $\beta$ versus $T^{-1}$ plot presented here, the relaxation is indeed slightly nonexponential at all temperatures.

\section{$\underline{\text { References }}$}

1 L. K. Runnells, Phys. Rev. 134 (1964) A28-A36.

2 R. L. Hilt and P. S. Hubbard, Phys. Rev. 134 (1964) A392-A398.

3 S. Emid and R. A. Wind, Chem. Phys. Lett. 27 (1974) 312-316.

4 A. Abragam, The Principles of Nuclear Magnetism (Oxford Univ. Press, Oxford, 1961).

5 N. Bloembergen, E. M. Purcell, and R. V. Pound, Phys. Rev. 73 (1948) 679-712.

6 R. K. Wangsness and F. Bloch, Phys. Rev. 89 (1953) 728-739.

7 I. Solomon, Phys. Rev. 99 (1955) 559-565.

8 F. Bloch, Phys. Rev. 102 (1956) 104-135.

9 P. S. Hubbard, Phys. Rev. 109 (1958) 1153-1158. Also, see a correction in P. S. Hubbard, Phys. Rev. 111 (1958) 1746-1747.

10 D. E. Woessner, J. Chem. Phys. 36 (1962) 1-4.

11 F. Bloch, Phys. Rev. 105 (1957) 1206-1222.

12 A. G. Redfield, IBM J. Res. Develop. 1 (1957) 19; reprinted with minor revisions in Advan. Mag. Resonan. 1 (1965) 1-32.

13 K. Tomita, Prog. Theor. Phys. 19 (1958) 541-580.

14 E. O. Stejskal and H. S. Gutowsky, J. Chem. Phys. 28 (1958) 388-396. See a correction in the footnote on page 973 of ref. 56.

15 C. P. Slichter, Principles of Magnetic Resonance (3rd ed., Springer-Verlag, Berlin, 1990).

16 R. R. Ernst, G. Bodenhausen, and A. Wokaum, Principles of Nuclear Magnetic Resonance in One and Two Dimensions (Oxford Univ. Press: Oxford, 1987). 
17 R. Kimmich, NMR Tomography, Diffusometry, Relaxometry (Springer-Verlag, Berlin, 1997).

18 M. Goldman, J. Mag. Resonan. 149 (2001) 160-187.

19 S. Clough and A. Heidemann, J. Phys. C: Solid State Phys. 13 (1980) 3585-3589.

20 S. Clough, A. Heidemann, A. J. Horsewill, J. D. Lewis, and M. N. J. Paley, J. Phys. C: Solid State Phys. 14 (1981) L525-L529.

21 S. Clough and P. J. McDonald, J. Phys. C: Solid State Phys. 15 (1982) L1039-L1042.

22 S. Clough, P. J. McDonald, and F. O. Zelaya, J. Phys. C: Solid State Phys. 17 (1984) 44134420.

23 D. Cavagnat, S. Clough, F. O. Zelaya, J. Phys. C: Solid State Phys. 18 (1985) 6457-6462.

24 S. Clough, Physica 136B (1986) 145-149.

25 M. J. Barlow, S. Clough, A. J. Horsewill, and M. A. Mohammed, Solid State Nuc. Mag. Resonan. 1 (1992) 197-204.

26 S. Clough, Solid State Nuc. Mag. Resonan. 9 (1997) 49-53.

27 J. S. Waugh and É. I. Fedin, Soviet Physics - Solid State, 4 (1963) 1633-1636.

28 R. Ferrando, R. Spadacini, G. E. Tommei, and V. I. Mel'nikov, Phys. Rev. E 51 (1995) R1645-R1648.

29 P. A. Beckmann, Molec. Phys. 34 (1977) 665-680.

30 J.-N. Dumez, P. Hakansson, S. Mamone, B. Meier, G. Stevanato, J. T. Hill-Cousins, S. S. Roy, R. C. D. Brown, G. Pileio, and M. H. Levitt, J. Chem. Phys. 142 (2015) 044506, 1-13.

31 M. Mehring and H. Raber, J. Chem. Phys. 59 (1973) 1116-1120.

32 M. Punkkinen, L. P. Ingman, D. G. Taylor, and P. S. Allen, J. Phys. C: Solid State Phys. 8 (1975) 2159-2166.

33 S. Emid and J. Smidt, Physica 121B (1983) 47-52.

34 M. F. Baud and P. S. Hubbard, Phys. Rev. 170 (1968) 384-390.

35 J. L. Carolan and T. A. Scott, J. Mag. Resonan. 2 (1970) 243-258.

36 K. van Putte, J. Mag. Resonan. 5 (1971) 367-375.

37 K. van Putte and G. J. N. Egmond, J. Mag. Resonan. 4 (1971) 236-240.

38 S. Albert and J. A. Ripmeester, J. Chem. Phys. 57 (1972) 2641-2645.

39 J. D. Cutnell and L. Verudian, J. Chem. Phys. 59 (1973) 258-262.

40 J. D. Cutnell and W. Venable, J. Chem. Phys. 60 (1974) 3795-3801.

41 E. R. Andrew, R. Gaspar Jr., and W. Vennart, Chem. Phys. Lett. 38 (1976) 41-43.

42 L. J. Burnett and B. H Muller, Chem. Phys. Lett. 18 (1973) 553-556.

43 P. S. Hubbard, J. Chem. Phys. 51 (1969) 1647-1651.

44 A. Kumar and C. S. Johnson, Jr., J. Chem. Phys. 60 (1974) 137-146. 
45 P. A. Beckmann, C. A. Buser, C. W. Mallory, F. B. Mallory, and J. Mosher, Solid State Nuc. Mag. Resonan. 12 (1998) 251-256.

46 T. Fukunaga, N. Kumagae, and H. Ishida, Z. Naturfor. A58 (2003) 631-637.

47 N. Pislewski, J. Tritt-Goc, M. Bielejewski, A. Rachocki, T. Ratajczyk, and S. Szymanski, Solid State Nuc. Mag. Resonan. 35 (2009) 194-200. \{The 's' in 'Pislewski' should have an acute accent.\}

48 C. Sun and A. J. Horsewill, Solid State Nuc. Mag. Resonan. 35 (2009) 139-146.

49 J. Klafter and M. F. Shlesinger, Proc. Natl. Acad. Sci. 83 (1986) 848-851.

50 J. Leise, K. Schmidt-Rohr, and H. W. Spiess, Physica A 201 (1993) 79-87.

51 R. G. Palmer, D. L. Stein, E. Abrahams, and P. W. Anderson, Phys. Rev. Lett. 53 (1984) 958961.

52 D. Wolf, J. Mag. Resonan. 17 (1975) 1-19.

53 W. A. Barton and C. A. Sholl, J. Phys. C: Solid State Phys. 9 (1976) 4315-4328.

54 J. Tegenfeld and R. Sjoblom, J. Mag. Resonan. 55 (1983) 372-377.

55 U. Haeberlen, Polymer 9 (1968) 50-52.

56 M. B. Dunn and C. A. McDowell, Molec. Phys. 24 (1972) 969-978.

57 K. G. Conn, P. A. Beckmann, C. W. Mallory, and F. B. Mallory, J. Chem. Phys. 87 (1987) 2027.

58 T. K. Jahnke, W. Müller-Warmuth, and M. Bennati, Solid State Nucl. Mag. Resonan. 4 (1995) 153-161.

59 P. A. Beckmann and E. Schneider, J. Chem. Phys. 136 (2012) 054508, 1-9.

60 E. K. van Putte, J. Mag. Resonan. 2 (1970) 216-225.

61 J. Kowaleski and T. Liljefors, Chem. Phys. Lett. 64 (1979) 170-174.

62 O. Edholm and C. Blomberg, Chem. Phys. 56 (1981) 9-14.

63 A. Detken, P, Focke, H. Zimmermann, U. Haeberlen, Z. Olenjinczak, and Z. T. Lalowicz, Z. Naturforch. A50 (1995) 95-116.

64 R. E. Taylor, P. A. Beckmann, S. Bai, and C. Dybowski, J. Phys. Chem. C 118 (2014) 91439153.

65 D. P. Fahey, W. G. Dougherty Jr., W. S. Kassel, X. Wang, and P. A. Beckmann, J. Phys. Chem. A 116 (2012) 11946-11956.

66 C. G. Van der Walle, Phys. Rev. B 53 (1996) 11292-11295.

67 R. Kohlrausch, Ann. Phys. Chem. (Poggendorff) 91 (1854) 179-214.

68 M. N. Berberan-Santos, E. N. Bodunov, and B. Valeur, Chem. Phys. 315 (2005) 171-182.

69 D. C. Johnson, Phys. Rev. B 74 (2006) 184430, 1-7.

70 M. Berberan-Santos, E. N. Bodunov, and B. Valeur, Ann. Phys. (Berlin) 17 (2008) 460-461. 
71 P. A. Beckmann, C. W. Mallory, F. B. Mallory, A. L. Rheingold, and X. Wang, ChemPhysChem 16 (2015) 1509-1519.

72 A. J. Horsewill, Prog. Nucl. Mag. Resonan. Spec. 35 (1999) 359-389.

73 F. B. Mallory, C. W. Mallory, K. G. Conn, and P. A. Beckmann, J. Phys. Chem. Sol. 51 (1990) 129-134.

74 P. A. Beckmann, C. A. Buser, K. Gullifer, F. B. Mallory, C. W. Mallory, G. M. Rossi, and A. L. Rheingold, J. Chem. Phys. 118 (2003) 11129-11138.

75 J. Haupt, Z. Naturforsch. A26 (1971) 1578-1589.

76 S. Clough, A. Heidemann, A. J. Horsewill, J. D. Lewis, and M. N. J. Paley, J. Phys. C: Solid State Phys. 15 (1982) 2495-2508.

77 U. Werner and W. Müller-Warmuth, Z. Physik B 91 (1993) 65-76.

78 G. Diezemann, App. Mag. Resonan. 17 (1999) 345-366.

79 X. Wang, P. A. Beckmann, C. W. Mallory, A. L. Rheingold, A. G. DiPasquale, P. J. Carroll, and F. B. Mallory, J. Org. Chem. 76 (2011) 5170-5176.

80 X. Wang, L. Rotkina, H. Su, and P. A. Beckmann, ChemPhysChem 13 (2012) 2082-2089.

81 X. Wang, A. L. Rheingold, A. G. DiPasquale, F. B. Mallory, C. W. Mallory, and P. A. Beckmann, J. Chem. Phys. 128 (2008) 124502, 1-3.

82 X. Wang, F. B. Mallory, C. W. Mallory, H. R. Odhner, and P. A. Beckmann, J. Chem. Phys. 140 (2014) 194304, 1-15.

83 P. A. Beckmann, A. M. Cheung, E. E. Fisch, F. A. Fusco, R. E. Herzog, and M. Narasimhan, J. Chem. Phys. 84 (1986) 1959-1968.

Table. Information for the six polycrystalline solids

\begin{tabular}{|c|l|c|c|c|}
\hline & name & $\begin{array}{c}\text { orig ref } \\
\text { for } R_{\text {ave }}\end{array}$ & $\begin{array}{c}\text { orig ref } \\
\text { for } R^{*} \& \beta\end{array}$ & $\begin{array}{c}E\left(\mathrm{~kJ} \mathrm{~mol}^{-1}\right) \\
\mathrm{CH}_{3} \text { rotation }\end{array}$ \\
\hline \hline $\mathbf{1}$ & 1- $\mathrm{CD}_{3}$, 9- $\mathrm{CH}_{3}$, phenanthrene & 73 & this work & $12(1)$ \\
\hline $\mathbf{2}$ & 1-methoxyphenanthrene & - & 71 & $16(2)$ \\
\hline $\mathbf{3}$ & 4,4'-dimethoxybiphenyl & - & 59 & $12(1)$ \\
\hline $\mathbf{4}$ & 3-ethylchrysene & 45 & this work & $12(1)$ \\
\hline $\mathbf{5}$ & 3-isopropylchrysene & 45 & this work & $11(1)$ \\
\hline $\mathbf{6}$ & 3-t-butylchrysene & 74 & this work & $24(2) / 14(1)^{\mathrm{a}}$ \\
\hline
\end{tabular}

${ }^{a}$ There are two distinct types of methyl groups in this $t$-butyl group. 


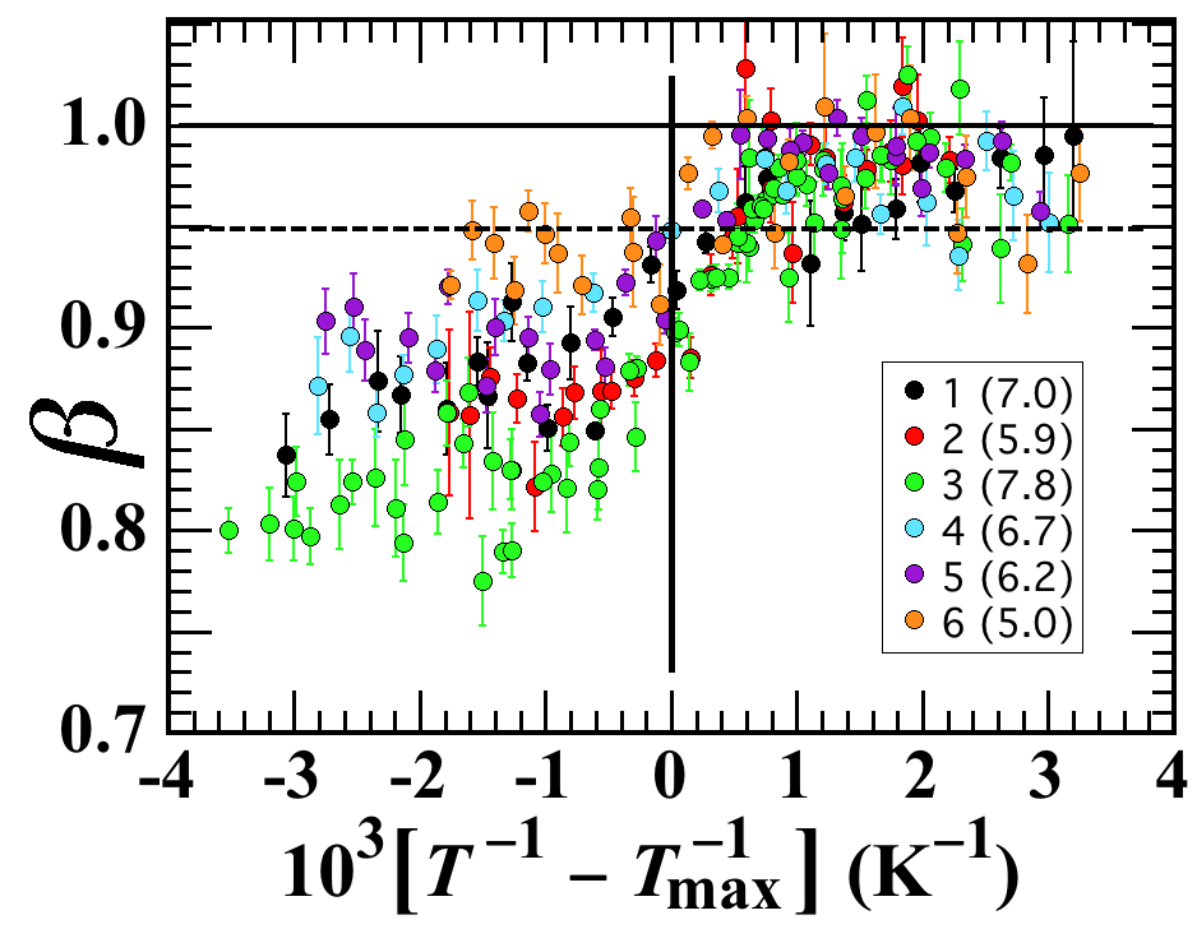

Figure. The stretching parameter $\beta$ in $M(t)=M(\infty)\left[1-(1-\cos \theta) \exp \left\{-\left(R^{*} t\right)^{\beta}\right\}\right]$ versus $T^{-1}-T^{-1}{ }_{\max }$ where $T$ is the temperature and $T_{\max }$ is the temperature of the relaxation rate maximum. The six compounds are identified in the table. The numbers in parentheses in the legend are the values of $10^{-3} T^{-1}$ max $\left(\right.$ in $\left.\mathrm{K}^{-1}\right)$. The data for $\mathbf{2}$ are taken from ref. [71] and the data for $\mathbf{3}$ are taken from ref. [59]. 